\title{
Dynamic Analysis and Verification of Structurally Optimized Nano-Satellite Systems
}

\author{
Srikanth Raviprasad ${ }^{1}$ and Nagaraj S Nayak ${ }^{2}$ \\ 1. Manipal Institute of Technology- Manipal University,Manipal 576104, India \\ 2. Mechanical and Industrial Engineering, Caledonian College of Engineering, Glasgow Caledonian University, CPO Seeb 111, \\ Sultanate of Oman.
}

\begin{abstract}
The need for humans to explore space is ever increasing with the complexity of problems encountered, requiring experiments at different physical conditions. The cost and other resources incurred in each of the space missions are soaring, irrespective of the entanglements of these missions. Nano-satellites have paved way in this regard, as they are cheap, feasible and provide a platform for new technologies to be tested and validated in space which can then be extended to larger satellites for more applications. Dynamic analysis of a structure is crucial in its conceptual design phase, so as to determine natural frequencies of the individual bodies and also the deformation and stress induced at the corresponding mode of vibration, and to ensure that the overall coupling frequency is well out of the danger zone, so as to avoid resulting structural damages. This paper deals with performing modal, harmonic and random vibration analysis of a structurally optimized nano-satellite and comparing this with the value of frequency with forcing function due to the launch vehicle loads obtained from experimental data from Indian Space Research Organization. The paper also provides a brief insight about different aspects considered like, structural constraints, deployer design, material selection, etc.
\end{abstract}

Key words: Nano-satellite structural designs, natural frequency, finite element methods, modal analysis, harmonic analysis, random vibration analysis.

\section{Introduction}

Nano-Satellite missions have become very frequent and it becomes imperative that the nano-satellite structure, just as any other satellite structure, is able to withstand the loads and forces acting on it during launch. Along with strength, the nano-satellite structure has to be designed under strict constraints imposed by other subsystems and due to the ejection mechanism that is conceived. It is due to this requirement that makes the selection of a structure design so hard. For most cases, the design is inspired by the pre-existing Cubesat designs developed by many commercial firms. FEM analysis of the structure is of major importance because any small issue could potentially lead to damage to the subsystem components or complete mission failure, hence it is

Corresponding author: Srikanth Raviprasad, research field: aerospace structures and materials, mechanical and manufacturing engineering. E-mail: srikanthraviprasad@gmail.com. mandatory for any space faring mission to be analysed and validated against the launch vehicle constraints to ensure maximum reliability.

The design and analysis of structures is considered the most basic requirement for any application and implementation of a concept or idea. Concerning satellite systems, this ideology becomes very critical as the structure is typically unrepairable once it is into an orbit.

A rigid structure is essential for any spacecraft to complete its mission. It has to be designed to act as containment for all subsystems and maintain structural integrity against axial loading, strain, torsion, and buckling. Smaller satellites are a current trend since they require less material and therefore cheaper. Therefore, a complete structural analysis is necessary to provide a pledge on the satellite structural reliability [1].

Miniaturized satellites or small satellites are artificial satellites of low mass and size, usually under 
$500 \mathrm{~kg}$ (1, $100 \mathrm{lb})$. One reason for miniaturizing satellites is to reduce the cost. In retrospect, smaller and lighter satellites require smaller and cheaper launch vehicles and can sometimes be launched in multiples. They can also be launched "piggyback", using excess capacity on larger launch vehicles. Miniaturized satellites allow for cheaper designs as well as ease of mass production. The term "nano-satellite" or "nano-sat" is applied to an artificial satellite with a wet mass between 1 and $10 \mathrm{~kg}$ (2.2 and $22 \mathrm{lb}$ ) [2].

Significant attempts have been made to standardize the nano-satellite analysis formulations, with different boundary conditions and assumptions to reduce the computational complexity. Moustafa Eiswy et al. [3] performed static and dynamic analysis for a 1 unit nano-satellite structure with its internal components integrated. The first longitudinal mode of vibration was obtained as $180.68 \mathrm{~Hz}$ which is greater than $100 \mathrm{~Hz}$, as specified in his work. The mention about contact conditions employed for the FEM analysis is missing in the paper. Also, a modal analysis performed to obtain the frequency at the minimum mode of vibration, is not validated with a harmonic analysis, which is quintessential. Use of bolted contacts in the work could have rendered it more rigid and integrated.

Hamid Khan, Asif Israr [4] performed the analysis of a low earth orbit satellite. Element used for FEM analysis was SOLID186. Modal analysis was performed and the value $82.033 \mathrm{~Hz}$ obtained was higher than the minimum first mode frequency of 80 Hz. Quiroz-Garfias et al. [5] performed the finite element analysis and design of a Cubesat class pico-satellite structure. Static and dynamic loads were applied based on the launch vehicle constraints, and the results obtained were consistent with the required values. This work was focused towards only the primary structure of the satellite, which basically is the outer skin of the satellite. The first mode of vibration obtained was $764.06 \mathrm{~Hz}$ for the external structure, which maybe favourable for the launch vehicle. But, this value is sure to drop drastically when the module is integrated with all the required internal components for a successful space mission.

The objective of this work is to consider a structurally optimized nano-satellite design, conduct modal analysis to determine its natural frequency and a harmonic analysis to make sure that the maximum amplitude of deformation obtained below the specified minimum frequency does not exceed the limiting value; and also a random vibration analysis to find the stresses due to vibration loads that are random in nature. Finally the results are compared with the experimentally obtained values of a particular launch vehicle, to check the integrity of the structure under those launch loads.

\subsection{Nanosatellite Design Concepts}

The Cubesat program [6] was started at Stanford University in early 1999 to meet an educational need to have a satellite that could be developed within one, two years, be very-low cost and be very low weight for reduced launch costs. Selecting the weight of a pico-satellite $(<1 \mathrm{~kg})$ as the starting basis and the size of a $10 \mathrm{~cm}$ cube a design was established and called the Cubesat.

Four basic primary structure design concepts were considered for Cubesat designs [7]. They include:

- Mono-block

- Double mono-block

- Structure in panels

- Multiple piece concept

After a detailed case study, an extensive structural analysis was performed in order to select the best possible structure. The multi-piece concept was considered as the most reliable and optimized primary structure for a Cubesat. This was concluded based on less magnitude of the Von-Mises stress levels obtained as compared to the other designs proposed. Certain other critical criteria like weight, ease of manufacturing, assembly constraints etc. were taken into account while deciding the same [8].

Fig. 1 illustrates multi piece design which includes 4 
$\mathrm{H}$ brackets or rails and 8 cross rails connecting them. These are connected using bolted joints for a robust structure.

The main advantages of this design are that all pieces can be fabricated from commonly available extruded sections; they do not require expensive machining and the design will allow for a short design time. The main disadvantage is that the external geometrical tolerances are difficult to reach. Moreover the mass of the frame is raised, because there are a lot of interfaces and thus many screws. Al 6061-T6 is considered most appropriate for a satellite structure. Also, the Cubesat Standard specifies that the satellite must be constructed of a material with a similar thermal expansion coefficient to the materials used for the construction of the P-POD. The P-POD has been fabricated from the aluminium alloy Al-7075-T73. The specifications recommend the use of Al-6061-T6 [7].

\section{Simulation of Nano-Satellite Systems}

\subsection{Methodology}

The methods adopted in the current study are presented here. Initially the constraints set on the structural subsystem are discussed along with launch loads. Detailed design methodology is introduced and on those principles a design and material selection is done.

\subsubsection{Modal Analysis}

Modal analysis is a process which is used to describe a structure in terms of its natural characteristics, which are the frequency, damping and mode shapes-its dynamic properties [9].

It is used to determine the vibration characteristics (natural frequencies and mode shapes) of a structure while it is being designed. When frequencies of loading vibrations match one of these natural frequencies, resonance takes place. For any dynamic structure, resonance is one of the most critical problems that control its design [10].

Fig. 2 shows the behavior of a flat plate under different loading conditions or forcing functions. Basically the dynamic characteristics depend on the

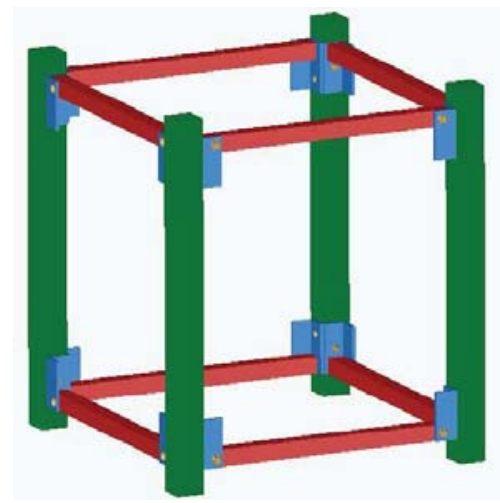

Fig. 1 Multi-piece concept [7].

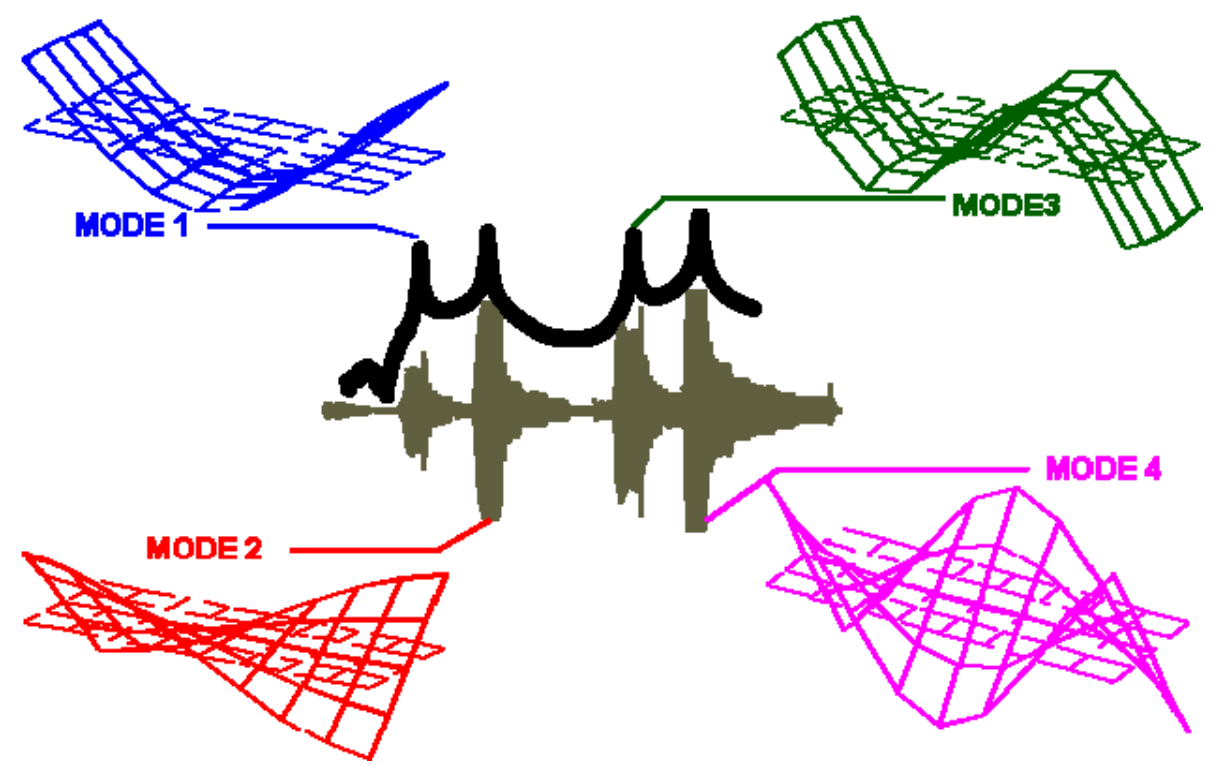

Fig. 2 Simple plate dwell responses [9]. 
weight and stiffness of the structure considered which determine where these natural frequencies and mode shapes will exist [9].

\subsubsection{Harmonic Analysis}

Harmonic analysis is a technique used to determine the steady-state response of linear structures to loads that vary in sinusoidal nature (harmonic) with time. In harmonic analysis, the entire structure has constant or frequency-dependent stiffness, damping and mass effects. Thereafter, peak responses are identified and stresses reviewed at those peak frequencies [11]. This analysis technique calculates only steady state forced vibration of a structure. The transient vibrations, which occur at the beginning of the excitation, are not accounted for in harmonic analysis [12].

Fig. 3 depicts the transient and steady state dynamic response of a structural system.

\subsubsection{Random Vibration Analysis}

Random vibration analysis enables one to determine the response of structures to vibration loads that are ransom in nature. The frequency content of the time history (spectrum) is captured along with the statistics and used as the load in the random vibration analysis. This spectrum, for historical reasons, is called PSD (power spectral density) which is the input to the analysis. In a random vibration analysis since the input excitations are statistical in nature, so are the output responses such as displacements, stresses, and so on [12].

PSD is a statistical measure defined as the limiting mean-square value of a random variable. Here, the instantaneous magnitudes of the response can be

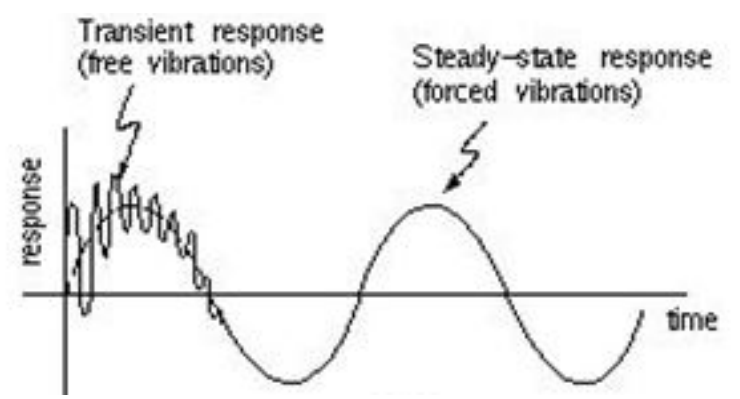

Fig. 3 Transient and steady state dynamic response of a structural system [12]. specified only by probability distribution functions that show the probability of the magnitude taking a particular value.

A PSD spectrum is a statistical measure of the response of a structure to random dynamic loading conditions. It is a graph of the PSD value versus frequency, where the PSD may be a displacement PSD, velocity PSD, acceleration PSD, or force PSD. Mathematically, the area under a PSD-versus-frequency curve is equal to the variance (square of the standard deviation of the response).

Similar to response spectrum analysis, a random vibration analysis may be single-point or multi-point. In a single-point random vibration analysis, one can specify one PSD spectrum at a set of points in the model. In a multi-point random vibration analysis, one can specify different PSD spectra at different points in the model. Fig. 4 clearly shows the single and multipoint response spectra depiction [12].

\subsection{Design Approach of Satellite Systems}

\subsubsection{Design Constraints [13]}

Since the purpose is to build a nano-satellite, the design specifications and constraints are considered as given by the Cubesat Standards. For the scope of this work, a 1 Unit Cubesat is considered. A I Unit Cubesat measures $10 \mathrm{~cm} \times 10 \mathrm{~cm} \times 10 \mathrm{~cm}$ along its dimensions. Among all the constraints which may be applicable to the structure of the satellite from all the subsystems, the basic deployer and launch load constraints are considered due to the absence of other subsystem data. The launch vehicle data considered for this report is that of PSLV (Polar Synchronous Launch Vehicle) provided by ISRO (Indian space research organization).

\subsubsection{Weight and Volume Constraints [13]}

The maximum weight of the satellite has been fixed to $1 \mathrm{~kg}$ based on the Cubesat standards being followed according to which a cuboid is sized $100 \times 100 \times 100$ $\mathrm{mm}^{3}$. The mass of the present model in the scope of this paper is $0.987 \mathrm{Kg}$. 


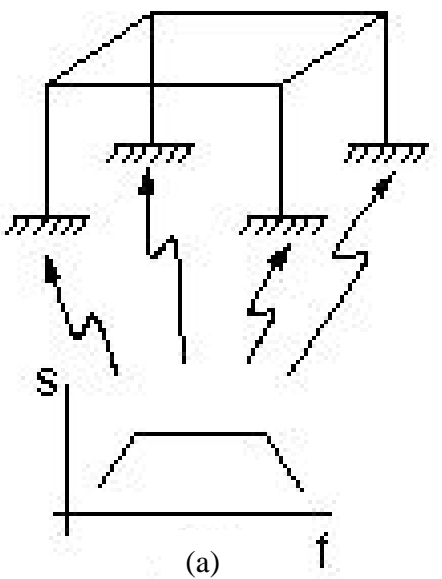

(a)

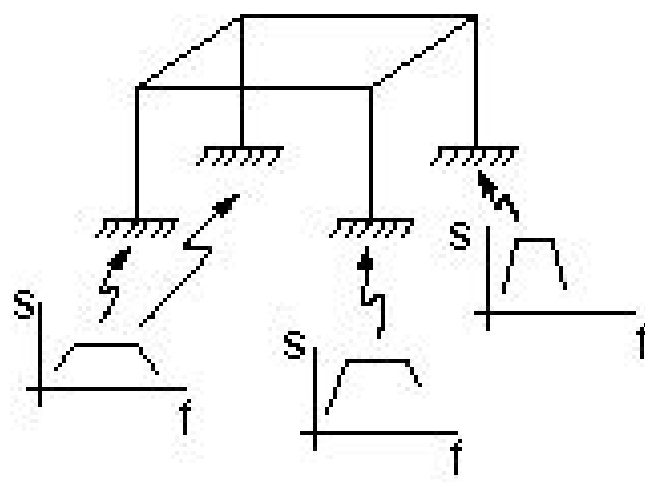

(b)

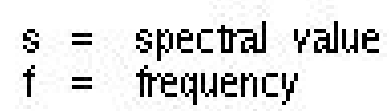

Fig. 4 Single-point and multi-point response spectra [12].

- The structure should not take more than $30 \%$ of the weight of the total satellite.

- The centre of mass of the satellite must be within $2 \mathrm{~cm}$ of the geometrical centre.

\subsubsection{Launch Load Requirements [13]}

According to the requirements set by the launch provider ISRO the satellite should be designed to take the loads specified during the launch as specified by PSLV documents. During the flight, the satellite is subjected to both inertial and dynamic loads. The design of the primary structure of satellite and mountings shall be verified for compliance with the following levels [14].

(1) Quasi-Static Loads

Both the loads are to be considered acting simultaneously through the centre of gravity of the satellite. In this work, the analysis is performed with the satellite being assumed to be placed such that its $\mathrm{X}$ axis (vector normal to the nadir face) is along the axis of the launch vehicle.

(2) Inertial Loads:

Along $\mathrm{X}$ axis of satellite: $\pm 11 \mathrm{~g}$

Along $Y$ axis of satellite: $\pm 6 \mathrm{~g}$

Along $\mathrm{Z}$ axis of satellite: $\pm 6 \mathrm{~g}$

A factor of safety of 1.5 has been considered for each of the above inertial loads.

A load factor is a dimensionless multiple of " $g$ " that represents the inertia force acting on a structure, so the sign of the load factor is opposite to that of the acceleration. Basically inertia force is the force that resists the net external force on an accelerating body. When a body is under steady state acceleration, with an unchanging applied force balanced by inertial loads, it is called quasi-static acceleration or inertial acceleration [15].

(3) Frequency Constraints

First axial mode: $>100 \mathrm{~Hz}$

First lateral mode: $>50 \mathrm{~Hz}$

\subsubsection{Deployer Constraints [13]}

The launch interface data to be used will be of the ISRO INLS (nano-satellite launch system) having closure of dimensions $100 \mathrm{~mm} \times 100 \mathrm{~mm} \times 300 \mathrm{~mm}$. It has four sliding surfaces on corners of $100 \mathrm{~mm} \times 100$ $\mathrm{mm}$ sides with a minimum $8 \mathrm{~mm}$ width for sliding during separation. It has four locators on each corners having concave recess of radius $3 \mathrm{~mm}$.

- The external components other than the rails shall not touch inside of the deployer.

- Components on sides shall not extend more than $6.5 \mathrm{~mm}$ normal to the surface.

At least $75 \%$ of the rail must be in contact with the deployer rails. $25 \%$ of the rails may be recessed and no part of the rails may exceed the specification [16]. Fig. 5 shows a deployer developed by ISRO. This deployer design is pre-defined for a $1 \mathrm{U}$ Cubesat, with the external dimensions of the deployer being $100 \times 100 \mathrm{~mm}$. 


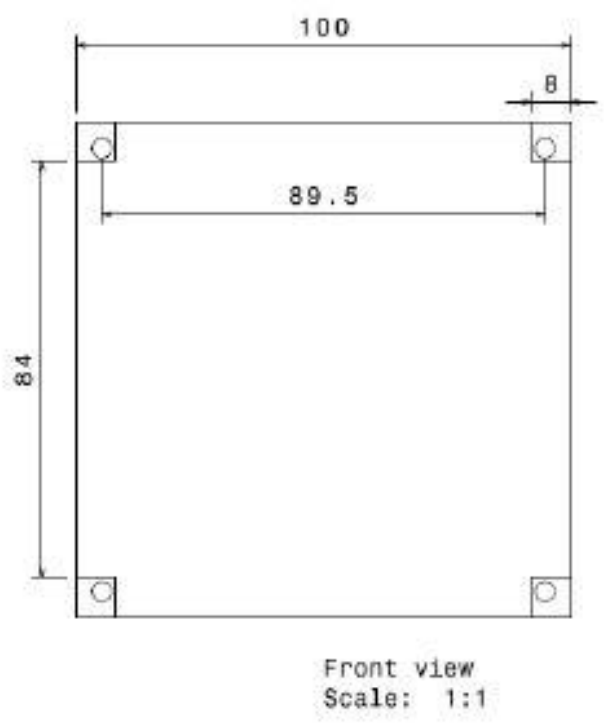

Fig. 5 Design sketch of deployer by INLS.

\section{Calculation Methodology}

\subsection{Analysis Setup}

The analyses were carried out on ANSYS V14.5 FEM software to find the required stresses and deformation under various loading conditions. The multi-piece design used here for the primary structure, is the optimized model of the Cubesat which was analysed for structural loads [8]. Internal components and skin of the satellite are mounted on this primary structure to get the assembled structure of the whole satellite.

\subsubsection{Joints}

Bolted screw joints contacts were considered for analysis. These screw joints experience a variety of loads resulting in corresponding stresses. Based on empirical studies, it has been considered that tensile stresses are predominant along the direction perpendicular to the cross section of the screws.

Bolted joints enable the contact between different parts of the Cubesat structure. Pretension of bolt is calculated with empirical related and is applied to the bolted joints. The material of screws selected for this purpose was Space graded SS304 (Stainless steel) M3 $\times 0.5 \mathrm{~mm}$ screws, and the calculated bolt pretension was $563.45 \mathrm{~N}$, as prescribed by the guidelines

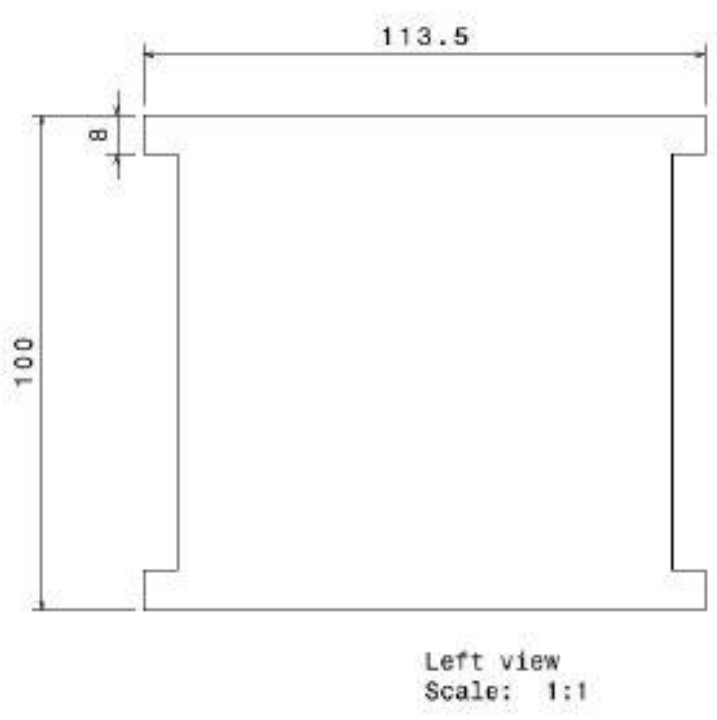

suggested by Budynas-Nisbett [17].

\subsubsection{Boundary Conditions}

The boundary conditions are applied for the analysis based on the launch vehicle's inertial load constraints.

Fig. 6 shows the isometric view of the assembled nano-satellite design before analysis and Fig. 7 shows the same without panels, so as to have an insight into the internal components of the nano-satellite. These figures are captured on ANSYS package and the pointers indicate positions of point mass considered each for the tertiary components in place.

The approach in these analyses is that all the major components of the satellite are place in the design and

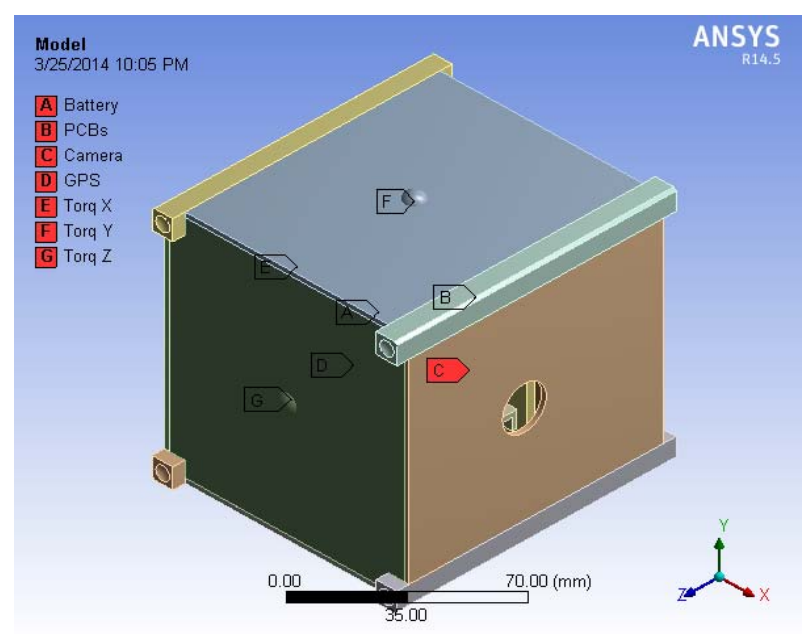

Fig. 6 Assembled Nano-satellite. 


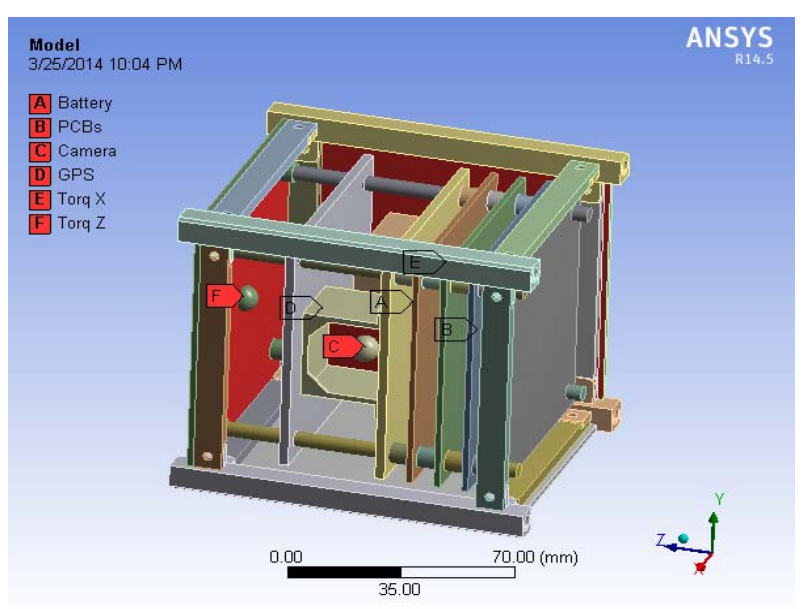

Fig. 7 Assembled Nano-satellite without panels.

then an analysis is done on the whole structure. The obtained results are compared to the worst possible actual case in the launch vehicle. Here, the launch vehicle data considered is that of PSLV of ISRO.

For each case, areas of contact of the H-Bracket are kept fixed corresponding to the direction in which the lateral forces are acting. Inertia forces are applied in terms of components on the primary structure.

A few tertiary components of the satellite, the imager (payload), Magnetorquers, batteries, etc were suppressed for simplification of computations involved in the simulation. To compensate the effect due to the above suppressed parts, point masses were considered at each of their centre of gravity positions. Contacts were defined with the mindset to achieve the most accurate condition representing the real life situation.

\subsubsection{Computational Domain}

The meshed model is shown in Fig. 8. The number of nodes in the domain was 3391971 and the number of elements obtained was 143005 .

The element shape is largely hexahedral throughout the structure, excepting for the edges and circular parts, where the free mesher automatically defines element shapes based on respective profiles and density of mesh. After setting a mesh relevance of 20 , and smooth transition formulation, the smart sizing tool sets the size of these elements in the most optimal way.

\subsubsection{Modal Analysis}

The modal analysis was performed to find the natural frequency of vibration of the integrated satellite system. The purpose of the analysis was to find, mainly the first mode of vibration and to verify that this frequency value obtained is higher than the specified minimum limit, in regard to the launch vehicle constraints.

The respective boundary conditions are applied for the analysis based on the launch vehicle's inertial load constraints. The constraints imposed by the launch vehicle were that the minimum frequency should be above $100 \mathrm{~Hz}$ along the axial direction of the launch vehicle and above $50 \mathrm{~Hz}$ in along the lateral direction. To account for maximum safety and reliability, $100 \mathrm{~Hz}$ has been considered as the minimum value along both the directions [14]. The Modal analysis was carried out in the absence of any external load.

\section{Boundary Conditions}

Fixed Supports: This type of support constraints the element in all directions and allows no deformations on the element. Fixed supports are applied to the 8 spherical recess bases of the 4 rails of the satellite.

Frictionless Supports: This type of support allows in plane motion but restricts motion normal to the plane. The basic idea is to simulate a physical contact while considering no friction between the 2 surfaces. The frictional component will be negligible between the satellite H-Bracket rails and the deployer rails. Frictionless support was chosen to simulate the contact

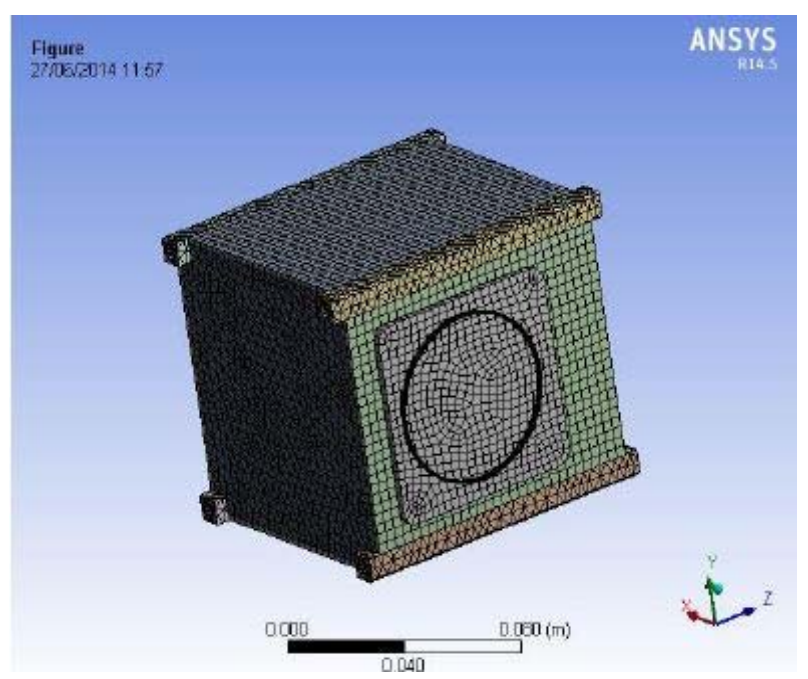

Fig. 8 Meshed model. 
faces between the satellite and the deployer while it is acted upon by the acceleration of the satellite.

Fig. 9 clearly illustrates the primary boundary conditions applied on the nano-satellite to be analyzed. The purple surfaces on rails indicate frictionless support and the ones on hemispherical stud depressions indicate fixed support boundary conditions in that region.

\subsubsection{Harmonic Analysis}

Harmonic analysis was performed in order to verify that the amplitude of vibration doesn't exceed the specified value (in meters) along the subsequent modes of vibration or excitation frequencies. This analysis carried out along two different directions with respect to the launch vehicle. The first one is along the longitudinal axis and the second one is along the lateral axis. The applied load conditions are given in Table 1 and 2.

Inertial acceleration value of $3.75 \mathrm{~g}$ was applied in the direction of the motion of the launch vehicle axis. The minimum frequency value considered was $10 \mathrm{~Hz}$ and the maximum considered was $200 \mathrm{~Hz}$ with 10 calculation sub-steps between them.

Inertial acceleration value of $2 \mathrm{~g}$ was applied in the direction perpendicular to the motion of the launch vehicle axis. The minimum frequency value considered was $10 \mathrm{~Hz}$ and the maximum considered was $200 \mathrm{~Hz}$ with 10 calculation sub-steps between them.

\subsubsection{Random Vibration Analysis}

Random vibration analysis was performed in order to verify that the deformation and stress amplitudes that occur instantaneously due to random unpredictable loads. The limit could either be the yield stress of the satellite material or a particular deformation value.

This analysis was carried out along the three global $\mathrm{X}, \mathrm{Y}$ and $\mathrm{Z}$ directions of the satellite system discreetly. The specified stress input data for the analysis was in the form of qualification PSD values in the units of $\mathrm{g}^{2} / \mathrm{Hz}$ corresponding to frequency input values as shown in Table 3.

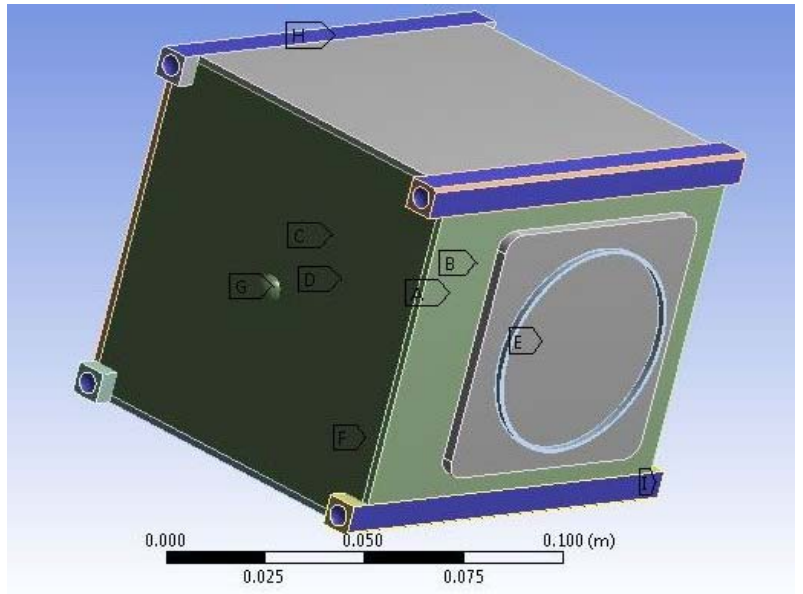

Fig. 9 Nano-satellite system-meshed model with modal analysis boundary conditions.

Table 1 Harmonic analysis loads along longitudinal axis [14].

\begin{tabular}{llll}
\hline & $\begin{array}{l}\text { Frequency } \\
\text { range }(\mathrm{Hz})\end{array}$ & $\begin{array}{l}\text { Qualification } \\
\text { level }\end{array}$ & $\begin{array}{l}\text { Acceptance } \\
\text { level }\end{array}$ \\
\hline $\begin{array}{l}\text { Longitudinal } \\
\text { axis load }\end{array}$ & $4-10$ & $\begin{array}{l}10 \mathrm{~mm} \\
(0-p e a k)\end{array}$ & $8 \mathrm{~mm}$ (0-peak) \\
$\begin{array}{l}\text { condition } \\
\text { Sweep rate }\end{array}$ & $10-100$ & $\begin{array}{l}3.75 \mathrm{~g} \\
2 \mathrm{Oct} / \text { minute }\end{array}$ & \begin{tabular}{l} 
4 Oct/minute \\
\hline
\end{tabular} \\
\hline
\end{tabular}

Table 2 Harmonic analysis loads along lateral axis [14].

\begin{tabular}{|c|c|c|c|}
\hline & $\begin{array}{l}\text { Frequency } \\
\text { range }(\mathrm{Hz})\end{array}$ & $\begin{array}{l}\text { Qualification } \\
\text { level }\end{array}$ & $\begin{array}{l}\text { Acceptance } \\
\text { level }\end{array}$ \\
\hline \multirow{2}{*}{$\begin{array}{l}\text { Lateral axis } \\
\text { load condition }\end{array}$} & $2-8$ & $\begin{array}{l}10 \mathrm{~mm} \\
\text { (0-peak) }\end{array}$ & $8 \mathrm{~mm}$ (0-peak) \\
\hline & $8-100$ & $2.25 \mathrm{~g}$ & $2 \mathrm{~g}$ \\
\hline Sweep rate & & 2 Oct/minute & 4 Oct/minute \\
\hline
\end{tabular}

Table 3 PSD values in used for simulations [14].

\begin{tabular}{lll}
\hline Frequency & $\begin{array}{l}\text { Qualification PSD } \\
\left(\mathrm{g}^{2} / \mathrm{Hz}\right)\end{array}$ & $\begin{array}{l}\text { Acceptance PSD } \\
\left(\mathrm{g}^{2} / \mathrm{Hz}\right)\end{array}$ \\
\hline 20 & 0.002 & 0.001 \\
110 & 0.002 & 0.001 \\
250 & 0.034 & 0.015 \\
1000 & 0.034 & 0.015 \\
2000 & 0.009 & 0.004 \\
gRMS & 6.7 & 4.47 \\
Duration & 2 min/axis & $1 \mathrm{~min} / \mathrm{axis}$ \\
\hline
\end{tabular}

The initial setup and pre-requisite data for Random Vibration analysis was directly coupled from the results of the modal analysis.

\section{Results and Discussion}

\subsection{Modal Analysis}

Free vibration occurs in the absence of, external 
excitation force. It is the result of some initial conditions imposed on the system, such as a displacement from the system's equilibrium position. Free vibration produces motion in one or more of the system's natural frequencies. Table 4 shows the values of natural frequency obtained for the first six modes of vibration. The present work aims to compare the minimum mode (first mode) frequency with the experimental data of the PSLV launch vehicle.

As clearly observed from Table 5 , the first mode of natural frequency occurs at $178.98 \mathrm{~Hz}$. This value obtained is well above the launch load minimum requirement of $100 \mathrm{~Hz}$ as stated and cited above. It can be deduced that the structure will not resonate with the launch vehicle interface and will survive the launch without any deformation. Hence, this model and internal assemble configuration can be considered as safe for a nano-satellite design. The deformation due to the first mode of vibration was observed on the PCBs (printed circuit boards) of the satellite. Hence, the material or the thickness of the PCB can be changed if needed, such that first mode of vibration occurs at much higher frequency than the minimum operating mode of the satellite assembly in the launch vehicle.

\subsection{Harmonic Analysis}

The harmonic analysis input frequency range and individual values are shown in Fig. 10. The first substep,

Table 4 Frequency values for different modes of vibration.

\begin{tabular}{ll}
\hline Mode & Frequency of vibration \\
\hline 1 & $178.98 \mathrm{~Hz}$ \\
2 & $310.90 \mathrm{~Hz}$ \\
3 & $311.07 \mathrm{~Hz}$ \\
4 & $311.43 \mathrm{~Hz}$ \\
5 & $475.25 \mathrm{~Hz}$ \\
6 & $475.71 \mathrm{~Hz}$ \\
\hline
\end{tabular}

Table 5 Results of maximum stress and deformation obtained in Random Vibration analysis.

\begin{tabular}{lll}
\hline $\begin{array}{l}\text { Maximum normal } \\
\begin{array}{l}\text { Stress } \\
(\mathrm{MPa})\end{array}\end{array}$ & $\begin{array}{l}\text { Percentage of yield } \\
\text { stress of Al 6061 T6 } \\
(\%)\end{array}$ & $\begin{array}{l}\text { Maximum } \\
\text { deformation } \\
(\mathrm{m})\end{array}$ \\
\hline 2.6 & 0.812 & $5.90 \mathrm{e}-06$ \\
\hline
\end{tabular}

being minimum, measures a frequency value of about $30 \mathrm{~Hz}$ and the 10th substep measures $200 \mathrm{~Hz}$.

Fig. 11 and 12 depict variation of the amplitude of deformation (in meters) and normal stress (in Pascals) with respect to frequency range, for longitudinal loading condition. A peak in amplitude is observed exactly corresponding to the frequency of first mode of vibration of the structure. Thus, it is consistent with the modal analysis performed previously in this paper. Moreover, the normal stress and deformation values obtained are extremely negligible when compared to the yield parameters of the structure, hence rendering it very safe.

Figs. 13 and 14 illustrate variation of the amplitude of deformation (in meters) and amplitude of normal stress (in Pascals) with respect to variation in frequency range, for lateral loading condition.

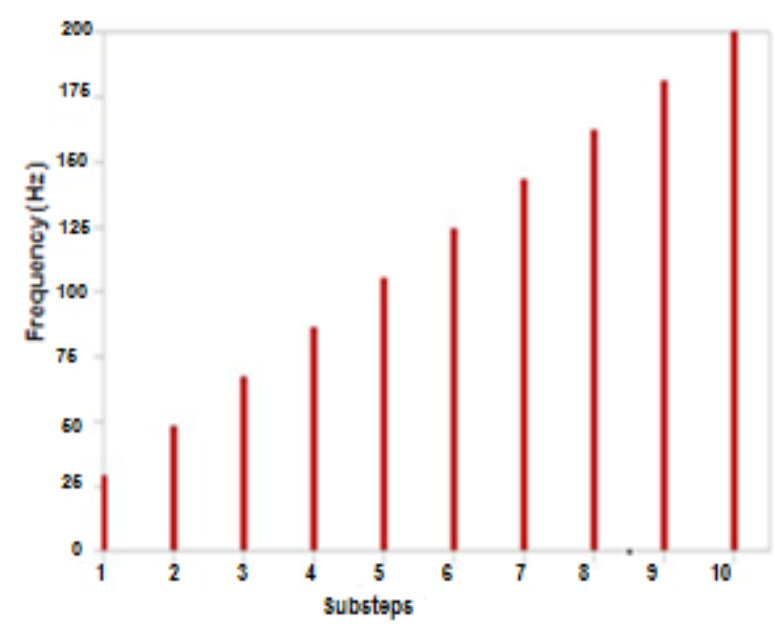

Fig. 10 Frequency values $(\mathrm{Hz})$ versus sub-steps.

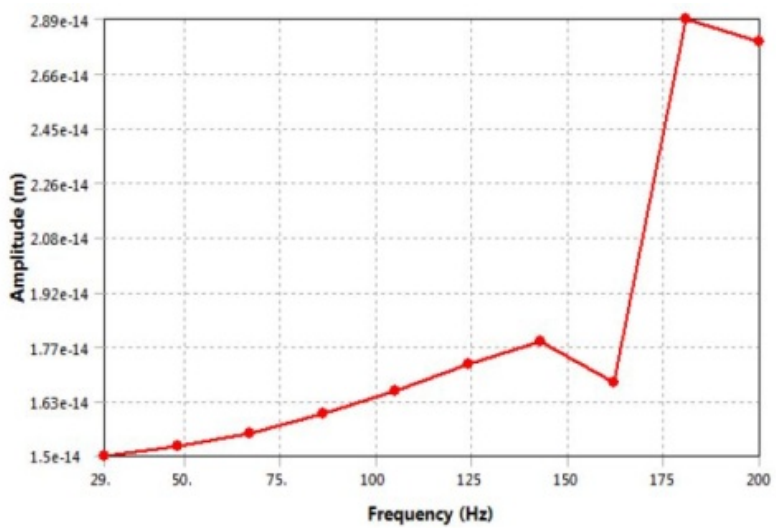

Fig. 11 Deformation amplitude (m) versus frequency ranges $(\mathrm{Hz})$ for longitudinal loading. 


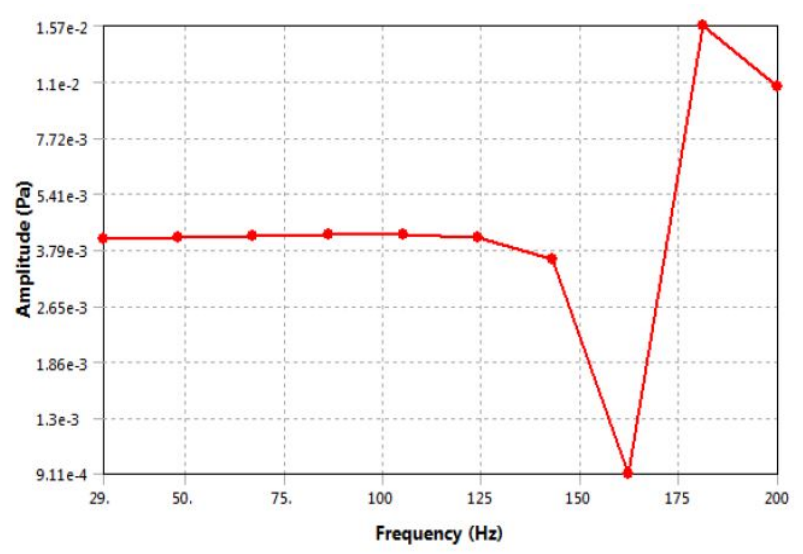

Fig. 12 Normal stress amplitude (Pa) versus frequency ranges $(\mathrm{Hz})$ for longitudinal loading.

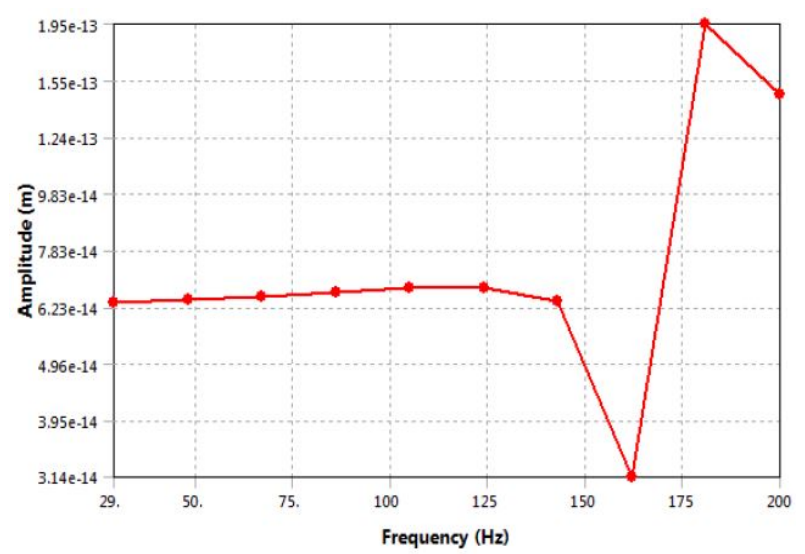

Fig. 13 Deformation amplitude (m) versus frequency ranges $(\mathrm{Hz})$ for lateral loading.

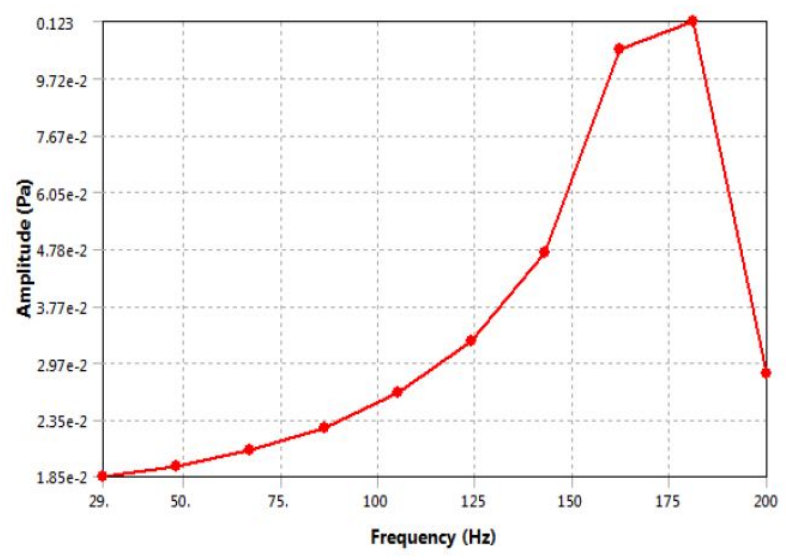

Fig. 14 Normal stress amplitude (Pa) versus frequency ranges $(\mathrm{Hz})$ for lateral loading.

From the figures, it is clear that a peak in amplitude observed is exactly corresponding to the frequency of first mode of vibration of the structure. Thus, it is consistent with the modal analysis performed previously in this work.

\subsection{Random Vibration Analysis}

The random vibration PSD input corresponding to the different frequency values is shown in Fig. 15.

The main objective of Random vibration analysis is to ensure that the stress levels and deformations incurred lies within permissible limits.

Table 5 contains the results of maximum stress and deformation obtained in random vibration analysis and Figs. 16 to 21 represent the magnitude of normal stresses as well as directional deformations. From the simulations, it can be concluded that, the maximum stress obtained is for the $\mathrm{X}$ direction case, i.e. stresses of 2.6 $\mathrm{MPa}$ is recorded and similarly the maximum deformation recorded is along the $\mathrm{Z}$ axis with a value of 5.9e-06 m which amounts up to 5.9 micrometer. On the contrary, the yield strength of Aluminium $6061 \mathrm{~T} 6$ is $320 \mathrm{MPa}$ which is 125 times larger than the stress incurred in the model.

Hence, it can be concluded that, the random vibrations caused during the different phases of space flight do not cause damage to the satellite system designed as the stresses and deformations are negligible when compared to the specified limits of operation.

\section{Conclusion}

This present work focuses on performing a complete dynamic analysis for a structurally optimized nano-satellite system. Initially, the natural frequency of excitation is determined by performing a modal

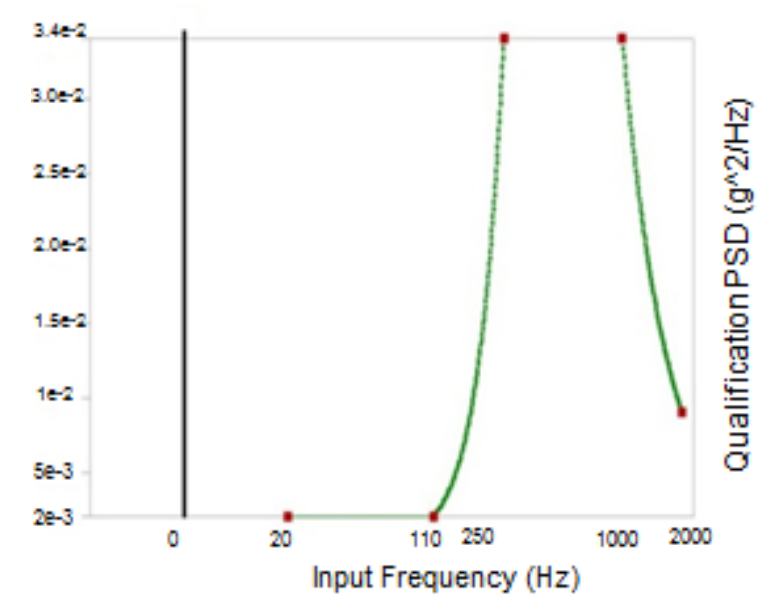

Fig. 15 Qualification PSD versus input frequency. 


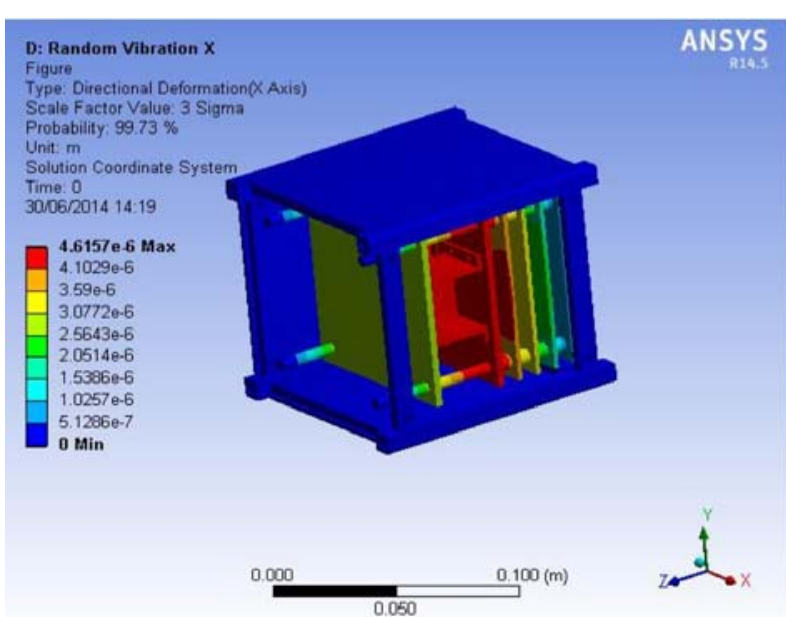

Fig. 16 Magnitude of deformation observed for random vibration along $X$ axis. The maximum value is observed on the battery box.

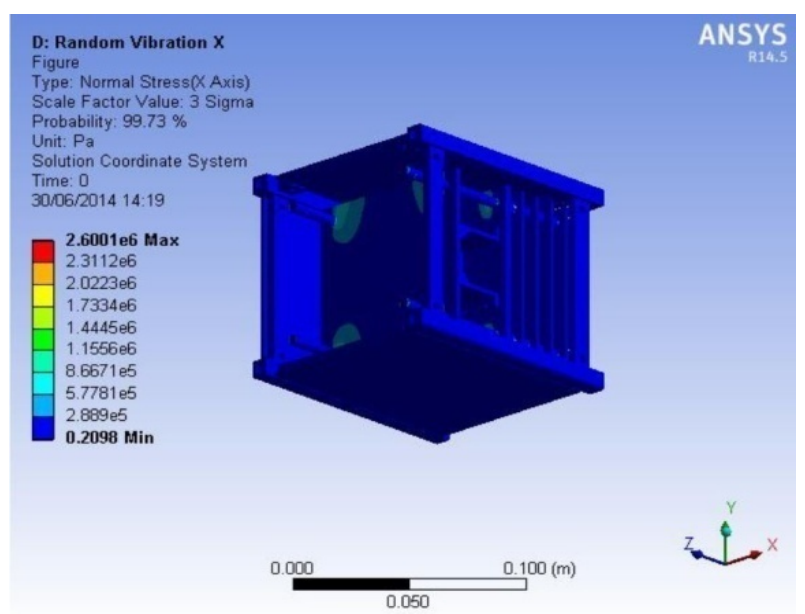

Fig. 17 Magnitude of normal stress observed for random vibration along $X$ axis, with maximum occurring in the interface between spacers and PCBs.

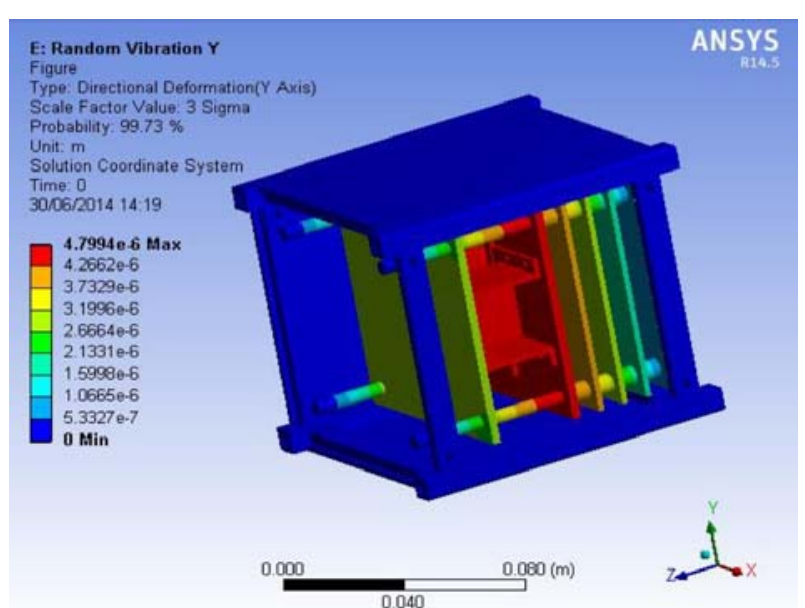

Fig. 18 Magnitude of deformation observed for random vibration along $Y$ axis. The maximum value is observed on the battery box and propagates down the PCB stack.

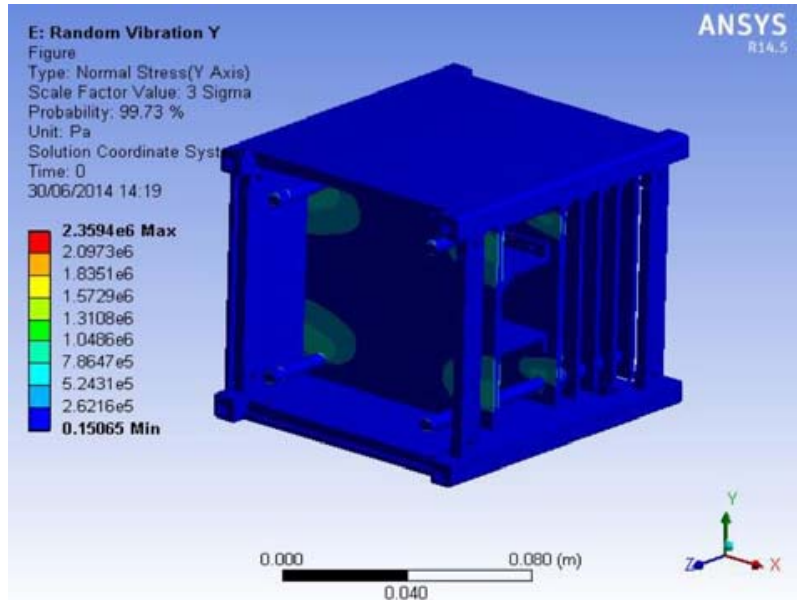

Fig. 19 Magnitude of Normal stress observed for random vibration along $\mathrm{Y}$ axis, with maximum occurring in the interface between spacers and PCBs.

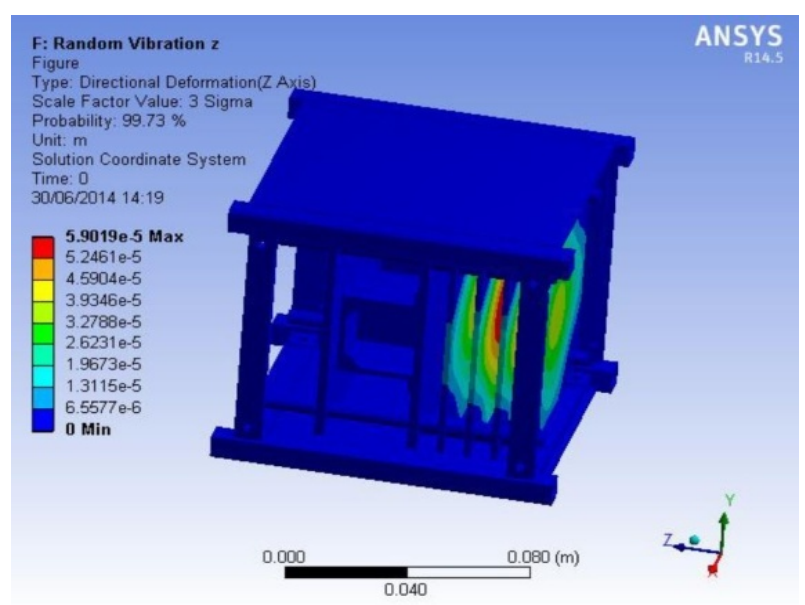

Fig. 20 Magnitude of deformation observed for random vibration along $Z$ axis. The maximum value of the same is observed in the centre of the PCB stack, precisely where mass concentration is highest.

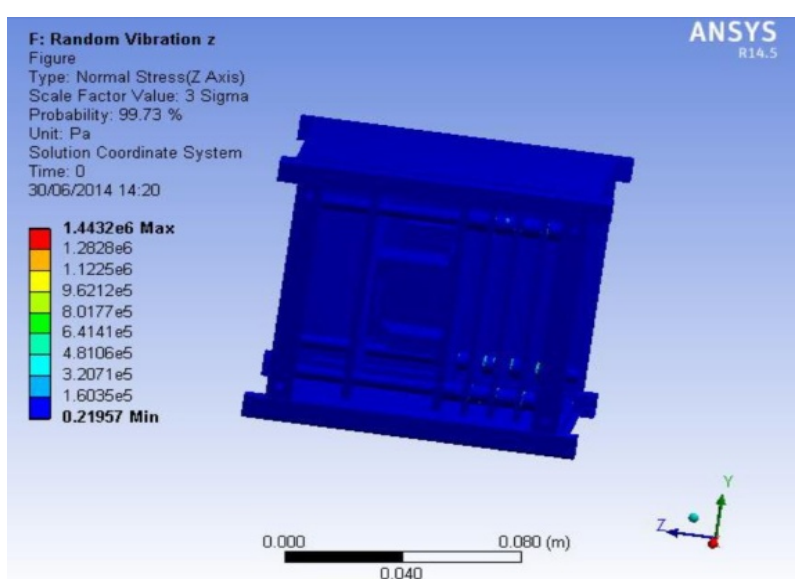

Fig. 21 Magnitude of normal stress observed for random vibration along $Z$ axis, with maximum occurring on the spacers. 
analysis with appropriate boundary conditions. Bolted joint contacts are considered in the present work. The value of the first mode excitation frequency obtained is compared with the minimum value of the frequency required for a spacecraft to vibrate, inside the launch vehicle in the launch phase. This minimum value differs slightly for different launch vehicles, the value being $100 \mathrm{~Hz}$ for PSLV of ISRO. The first mode of frequency occurred at $178.98 \mathrm{~Hz}$ which is well above $100 \mathrm{~Hz}$ limit. Hence, it can be safely concluded that the structural integrity will be maintained during the launch acceleration phase.

In addition to modal analysis, a harmonic analysis is performed with loading in two different cases, first being longitudinal and second being lateral, based on the given loads. The analysis results depicted that the normal stress as well as deformation values obtained are far lesser compared to the yield parameters of the material. As the maximum deformation occurred in both the cases at the frequency corresponding to the first mode of vibration, it was concluded that harmonic analysis is consistent with the modal analysis, hence rendering a safe design.

Finally, to predict the behaviour of the structure under random and uncontrolled loading conditions, a random vibration analysis was carried out on the structure. Again, the values maximum normal stress and deformation along each direction was very less when compared to the yield parameters of the material, resulting in the inference that these random loads would not affect the integrity of the structure at any phase of operation.

\section{Design Guidelines of Cubesats}

Cubesats are classified based on their dimensions as 1 Unit (1U), 2 Unit and 3 Unit nano-satellites. The maximum allowable mass of each of these three designs are $1.33 \mathrm{Kg}, 2.66 \mathrm{Kg}$ and $4 \mathrm{Kg}$. Also, centre of gravity of the satellite should be located within a sphere of $2 \mathrm{~cm}$ from its geometrical centre. The Cubesat is made up of Aluminium alloy Al6061-T6 and the deployer for ejection of the Cubesat is made up of Al7075-T75 alloy. This is to make sure that the coefficient of thermal expansion of the Cubesat and the deployer is almost the same. The joints and screws used to integrate different parts of the Cubesat are made up of stainless steel alloy SS304. Moreover, at least $75 \%$ of the area of the rails of the Cubesat must be in sliding contact with the rails of the deployer, to make sure that the ejection takes place smoothly [18]. Swiss cube had come up with different design concepts for the primary structure of a Cubesat, which have been structurally analysed by Srikanth Raviprasad et al. [8]. The Multi-piece primary structure configuration was found to be the best design based on the results obtained from the analysis.

\section{Acknowledgements}

The authors are thankful to the computational facilities provided by the CAD lab, Mechanical and Manufacturing Engineering department, Manipal Institute of Technology as well as the computational facilities provided by JNCASR (Jawaharlal Nehru Center for Advanced Scientific Research) for this research.

\section{References}

[1] Mojica, M. A. 2012. "Structural Subsystem Design, Analysis and Optimization for a Nanosatellite." MS Dissertation, San José State University, Department of Mechanical and Aerospace Engineering, San Jose CA.

[2] Stevens, C. L. 2002 "Design, Analysis, Fabrication and Testing of a Nanosatellite Structure" MS Dissertation, Virginia Polytechnic Institute and State University, Department of Aerospace Engineering, VA.

[3] Moustafa. E., Abdul, R. El S., Zafar, M., Hesham, H. I., and Tarek, N. 2012. "Emirates Aviation College Cubesat Project: Tuning of Natural Modes, Static and Dynamic Analyses of the Strength Model." 5th International Conference on RAST (Recent Advances in Space Technologies), Istanbul, doi: 0.1109/RAST.2011.5966842.

[4] Hamid, K., and Asif, I. 2011. "Design, Modeling and Analysis of Low Earth Orbit Satellite.” The Second TSME International Conference on Mechanical Engineering, Krabi

[5] Quiroz-Garfias, C., Silva-Navarro, G., and Rodriguez-Cortes, H. 2007. "Finite Element Analysis and Design of a Cubesat Class Picosatellite Structure." 4th International Conference on Electrical and Electronics 
Engineering, Mexico City.

[6] Puig-Suari, J., Clark, T., and Robert, J. T. 2001. “Cubesat: The Development and Launch Support Infrastructure for Eighteen Different Satellite Customers on One Launch.” Proceedings of the AIAA/USU Conference on Small Satellites, SSC01-VIIIb-5.

[7] Arce, D., Jutzeler, B., and Rothlisberger, G. 2006. "Swiss Cube: Phase A, Structure and Configuration.” École polyte-chnique fédérale de Lausanne, Lausanne Switzerland.

[8] Raviprasad, S., Vardhan, G. Y., and Nayyar, M. 2014. "Design and Analysis of different Nano-satellite Structures." Proc. International Conference for Environment and Sustainable Technologies, Manipal, India.

[9] Peter, A. 2001. "Experimental Modal Analysis: A Simple non Mathematical Presentation." University of Massachusetts Lowell, Lowell, Massachusetts.

[10] Gasser, F. A., Nader, A., and Ahmed, H. G. 2013. "Finite Element Analysis for Satellite Structures: Applications to Their Design, Manufacture and Testing.” Springer.

[11] Mohamed S. 2013 "ANSYS: Base Acceleration in
Harmonic Analysis-3 Techniques and 1 ACT Extension.” Internet: www.ansys-blog.com/harmonic-analysis/

[12] ANSYS, Inc. 2012. "Workbench User Guide”, Release 14.5, Canonsburg, PA.

[13] Anshuman, S., Noel, A., Prateek, N., and Rajat, S. 2011. "Parikshit Student Satellite: Conceptual Review:Structures and Mechanisms.” Manipal University.

[14] Guidelines for Student Satellite Proposal, 2011. Rev No. 1.0 Doc. No. ISRO-ISAC-TR-1036 [Report]/auth. Bangalore ISAC, India.

[15] Thomas, P. S., and Wiley, J. L. 1995. "Spacecraft Structures and Mechanisms-From Concept to Launch”, Torrance, California, Microcosm, Inc. ISBN 978-1881883036

[16] Guillaume Roethlisberger, 2008. "Swiss Cube: Phase C, Mechanical and Thermal ICD." École polyte-chnique fédérale de Lausanne, Lausanne Switzerland.

[17] Nisbett, B. 2008. “Shingley's Mechanical Engineering Design, Eighth Edition.” Tata McGraw-Hill Publication. ISBN-0-390-76487-6

[18] Cubesat Design Specification Rev. 13. 2014. The Cubesat Program, Cal Poly SLO. 